\title{
Theoretical Study of Photo-Luminescence Emission Using the Line Shape Function for Semiconductor Quantum Dots
}

\author{
P. Hari Krishna ${ }^{1, *}$, Devaanshi Jagwani², Meera Ramrakhiani ${ }^{3, \dagger}$ \\ ${ }^{1}$ Department of Physics, Medi-Caps University, Indore - 453331 (M.P.), India \\ 2 Department of Civil Engineering, IPS Academy, IES, Indore - 452012 (M.P.), India \\ ${ }^{3}$ Department of Post-Graduate Studies in Physics and Electronics, Rani Durgawati University, \\ Jabalpur - 482001(M.P.), India
}

(Received 10 January 2021; revised manuscript received 14 February 2021; published online 25 February 2021)

\begin{abstract}
The outcome of particle size effect on Photoluminescence has been investigated theoretically for CdS, $\mathrm{ZnS}$, CdSe and ZnSe quantum dots. The theory is based on computational modeling in the strong confinement region only where the particle size is less than Bohr's radius. The Photoluminescence emission line shape function which depends on band gap was found to be strongly dependent on particle size. The PL spectra of bulk material is similar for the quantum dots where similar vibronic coupling and normalized intensity is considered, only the peak wavelength changes and thus shifts the band edge luminescence peak to higher energies for the quantum dots corresponding to their band gap with no broadening. The PL spectra of monodisperse dots revels the fact that the shape of the emission peak is same, only it shift towards higher energy or smaller wavelength for decreasing size of quantum dots.
\end{abstract}

Keywords: Effective mass approximation, Quantization, Line shape function, Exciton bohr radius.

\section{INTRODUCTION}

Quantum dots are semiconductor nanocrystals in the range of 1-10 $\mathrm{nm}$ with sizes smaller than the Bohr exciton radius due to which quantum size effect in semiconductor QDs occur. It forms a zero dimensional semiconducting nanocrystal so called as dot [1]. Its optical and electronic behavior in this size regime lies between molecular and bulk form of matter. They possess very unique dimension dependent properties, such as size dependent band gap which leads to control and tune material's properties. The main advantage with QDs is the ability to tune the size of the dots for many applications [2]. In past years, a variety of quantum dots have been studied theoretically and experimentally also it has been found that the optical properties change dramatically from their bulk counterparts [3].

PL spectroscopy is a technique which can yield information on optical properties of nano semiconductors. The most easily observable effect through PL is the effect of monodisperse particles on the blue shift of optical absorption and emission of the semiconductor quantum dot with decreasing size due to the increase in band gap [4]. Different theoretical models have been developed for the determination of band structure parameters in semiconductor quantum dots. Among them, effective mass approximation model is most important and used in present theoretical investigation. The II-VI direct band gap semiconductors have gained substantial consideration among other groups due to their wide range of applications [5-7]. Present studies deals with intrinsic luminescence of such direct band gap materials. Modeling of variation of band gap with particle size and effect on their PL line shape function

\footnotetext{
*puranam.krishna@gmail.com

† mramrakhiani@hotmail.com

The results were presented at the International Conference on Multifunctional Nanomaterials (ICMN2020)
}

for CdS, ZnS, CdSe and ZnSe is described theoretically here using the concepts of condense matter physics, quantum mechanics and MATLAB programming.

\section{THEORETICAL MODELING}

\subsection{Variation of Band Gap with Particle Size}

Brus et al [8] considered the bulk materials, where the electron-hole pair is free to move in small quantum dots, become physically confined. Strong confinement leads to a rising of the electronic energy in the same way as would be expected from the simple particle-in-a-box model of quantum mechanics. Based on the effective mass approximation, the energy required for transition for such a system is given by the following equation [9].

$$
\begin{aligned}
& E\left(R_{0}\right)=E_{0}+\frac{\hbar^{2} \pi^{2}}{2}\left(\frac{1}{m_{e}^{*}}+\frac{1}{m_{h}^{*}}\right) \frac{1}{R_{0}^{2}}-\frac{1.8 e^{2}}{\varepsilon_{2}} \frac{1}{R_{0}}+ \\
& +\frac{e^{2}}{R_{0}} \sum_{n=1}^{\infty} \alpha_{0}\left(\frac{S}{R_{0}}\right)^{2 n}
\end{aligned}
$$

where, $E\left(R_{0}\right)$ is the effective band gap energy between valence band edge and conduction band edge of $Q$-dots, $E_{0}$ is the band gap of the bulk semiconductor, $\hbar=h / 2 \pi$ and $h$ is Planck's constant, $m^{*}{ }_{e}$ is the effective mass of electron and $m^{*} h$ is the effective mass of hole, $R_{0}$ is the radius of the nanoparticles with actual dielectric constant $\varepsilon_{2}=4 \pi \varepsilon_{0} \varepsilon_{r}$, where $\varepsilon_{r}$ is relative permittivity of medium, $\varepsilon_{0}$ permittivity of free space and $4 \pi \varepsilon_{0}=10^{7} / \mathrm{c}^{2}$, $\alpha_{0}$ is a function of dielectric constant and $\mathrm{S}$ is electronhole separation. The second term of this equation is the kinetic energy of electron and hole, the third term arises due to the Coulombic attraction between the 
electron and the hole and the last term is polarization energy which is neglected because in isotropic material symmetry reduces it to zero and also its calculation is not straight forward. Hence the equation 1 becomes

$$
E\left(R_{0}\right)=E_{0}+\frac{\hbar^{2} \pi^{2}}{2}\left(\frac{1}{m_{e}^{*}}+\frac{1}{m_{h}^{*}}\right) \frac{1}{R_{0}^{2}}-\frac{1.8 e^{2}}{\varepsilon_{2}} \frac{1}{R_{0}}
$$

It is important to note, while calculating $E\left(R_{0}\right)$ in $\mathrm{eV}$, the second term and third term must be converted to $\mathrm{eV}$ too. Actually, above equation is the direct solution to the Schrodinger equation for particle in one dimensional box, where a particle is subjected to stepped potential energy function infinite everywhere except for a finite line segment of length $L$.

\subsection{Expression for the Line Shape for Various Bulk Materials}

As seen from equation 1 the effective band gap varies with the size of nanoparticles which affects absorption as well as emission spectra of the materials. Thus the optical properties are strongly dependent on particle size, so considering the photoluminescence arising from the recombination of carriers at the band-edges in the bulk of direct-gap semiconductor with band-gap for bulk as $E_{0}$, the resulting line shape can be represented by a Gaussian profile [10]:

$$
g_{b}(E)=\frac{A}{\Gamma \sqrt{2 \pi}} \exp \left[-\frac{\left(E-E_{0}\right)^{2}}{2 \Gamma^{2}}\right],
$$

where $\Gamma$ is the parameter representing the intrinsic line width of the PL spectrum arising from the vibronic coupling. The full width at half maximum (FWHM) of Gaussian profile is equal to $2.354 \Gamma$. The prefactor ' $A$ ' represent the area under the curve and hence the integrated intensity. Thus the PL emission line shape is function of band gap $E_{0}$. Using MATLAB this has been plotted for four different luminescent materials CdS, $\mathrm{ZnS}, \mathrm{ZnSe}$ and CdSe. The parameter $\Gamma$ representing intrinsic line width of PL spectrum arising due to vibronic coupling is considered to be 0.1 [10]. The value of prefactor A has been taken by normalizing the PL intensity maximum at unity $i e$

$$
\frac{\mathrm{A}}{\Gamma \sqrt{2 \pi}} \exp \left[-\frac{\left(E-E_{0}\right)^{2}}{2 \Gamma^{2}}\right]=1 \text { Hence } A=\Gamma \sqrt{2 \pi}=0.25
$$

For CdS bulk band gap $E_{0}=2.43 \mathrm{eV}$ [11] therefore the line shape of bulk CdS material will be

$$
g_{b}(E)=\frac{0.25}{0.1 \sqrt{2 \times 3.14}} \exp \left[-\frac{(E-2.43)^{2}}{2(0.1)^{2}}\right]
$$

Considering the same values of $\Gamma, A$ and bulk band gap $E_{0}=3.68 \mathrm{eV}, 2.81 \mathrm{eV}$ and $1.71 \mathrm{eV}$ [11] the line shape of bulk $\mathrm{ZnS}, \mathrm{ZnSe}$ and CdSe material will be given by equations 5,6 and 7 respectively.

$$
g_{b}(E)=\frac{0.25}{0.1 \sqrt{2 X 3.14}} \exp \left[-\frac{(E-3.68)^{2}}{2(0.1)^{2}}\right]
$$

$$
\begin{aligned}
& g_{b}(E)=\frac{0.25}{0.1 \sqrt{2 X 3.14}} \exp \left[-\frac{(E-2.81)^{2}}{2(0.1)^{2}}\right] \\
& g_{b}(E)=\frac{0.25}{0.1 \sqrt{2 X 3.14}} \exp \left[-\frac{(E-1.71)^{2}}{2(0.1)^{2}}\right]
\end{aligned}
$$

Now $g_{b}(E)$ was calculated and plotted for different values of $E$ for different bulk materials using MATLAB modeling as shown in Figure 1.

\subsection{The Line Shape for CdS and $\mathrm{ZnS}$ Mono- disperse Nanoparticles}

Due to quantum confinement effect, the electronic energy levels of a semi-conductor nano-particle (quantum dots) are shifted with respect to bulk. The lowest direct inter-band transition energy of a spherical quantum dot of radius $R_{0}$ has been obtained by Equation 2 . If all the particles are of radius $R_{0}$, each one will have effective band gap $E\left(R_{0}\right)$ and therefore PL line shape can be obtained by simply substituting $E\left(R_{0}\right)$ in place of $E_{0}$ in Equation 3. The line shape for nanoparticles of radius $R_{0}$ can thus be given by [10]:

$$
g_{q b}\left(E, R_{0}\right)=\frac{A}{\Gamma \sqrt{2 \pi}} \exp \left[-\frac{\left(E-E\left(R_{0}\right)\right)^{2}}{2 \Gamma^{2}}\right]
$$

The parameters and constants for $\mathrm{CdS}$ has been taken as $E_{0}=2.43 \mathrm{eV}, m^{*}{ }_{e}=0.19 m_{0}$ [12], $m^{*}{ }_{h}=0.8 m_{0}$ [12-14], $\quad \varepsilon 2=5.7 \times 10^{7} / \mathrm{c}^{2}$ [12-14], for $\mathrm{ZnS} E_{0}=3.68 \mathrm{eV}$ $[15-17], \quad m^{*}{ }_{e}=0.41 m_{0}, \quad m^{*}{ }_{h}=0.61 m_{0}, \quad \varepsilon_{2}=8.3 \times 10^{7} / \mathrm{c}^{2}$ [15-17] substituting the above values in Equation 2 we get

$$
\begin{aligned}
& E\left(R_{0}\right)=2.43+\frac{2.44}{R_{0}^{2}}-\frac{0.4547}{R_{0}} \mathrm{CdS} \\
& E\left(R_{0}\right)=3.68+\frac{3.31}{\left(R_{0}\right)^{2}}-\frac{0.312}{R_{0}} \mathrm{ZnS}
\end{aligned}
$$

Depending upon the crystalline size of semiconductor nanocrystals, different type of confinements can be realized. When the radius of the crystallite $R_{0}$ is smaller than $\sim 2$ exciton Bohr radii, electrons and holes are considered as two confined particles, bound by an enforced Coulomb interaction and when the crystalline radius is larger than $\sim 4$ exciton radii, the ground exciton is treated as a rigid sphere, confined as a quasiparticle[18]. In between these two limiting cases both the electron and hole confinement and their Coulomb interaction are considered. The effective bandgap for various particle radius have been computed using equation 2 for CdS and $\mathrm{ZnS}$. It can be easily seen, for large values of $R_{0}$ (i.e. bulk) second and third term of Equation 2 reduces to zero and $E\left(R_{0}\right)=E_{0}$. For smaller values of $R_{0}$ (i.e. quantum dots) the kinetic energy of electrons and holes (second term) increases the effective band gap and the coulomb interaction energy (third term) reduces it. The effective band gap, the kinetic energy and the coulomb energy have been computed separately for CdS and ZnS quantum dots as shown in Table 1 and Table 2. Further using Equation 8 the PL 
line shape $g_{q d}\left(E, R_{0}\right)$ for different value of $E$ was calculated and plotted by modeling a program in the range $R_{0}$ in accordance with Table 1 and Table 2 . The plot between PL line shape (normalized intensity) vs photon energy are shown in Figure 2 and Figure 3 for monodisperse $\mathrm{CdS}$ and $\mathrm{ZnS}$ quantum dots.

\section{RESULTS}

The normalized PL intensity vs photon energy plot is shown in Figure 1 for different bulk materials. All the lines are similar since similar values of $A$ and $\Gamma$ has been taken. Only the peak position changes due to different values of band gap $E_{0}$ for various materials. The peak is exactly at the $E_{0}$ value which is clear from the Equation 3 (4 to 7 also). The corresponding wavelengths are 510, 337, 441 and $724 \mathrm{~nm}$ for $\mathrm{Cds}, \mathrm{ZnS}, \mathrm{ZnSe}$ and CdSe respectively.

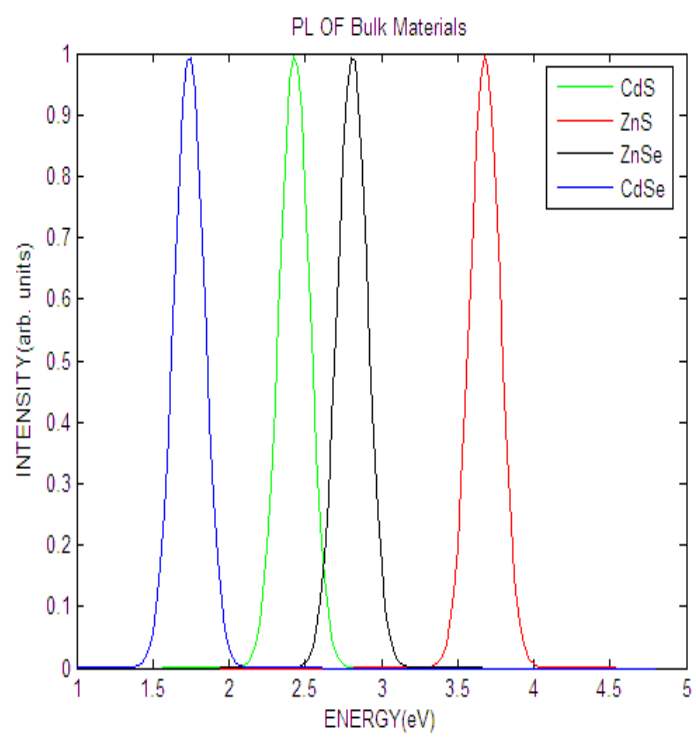

Fig. 1 - The normalized PL intensity vs photon energy

The calculated values of Effective band Gap, Kinetic energy and Coulomb energy for various sizes of CdS, and $\mathrm{ZnS}$ quantum dots are shown in Table 1 and Table 2 , as particle size is increased the effective band gap decreases first rapidly and then slowly further tends to become constant at the bulk band gap value. Above $5 \mathrm{~nm}$ and $4 \mathrm{~nm}$ quantization effect may not be observed in $\mathrm{CdS}$ and $\mathrm{ZnS}$ nanoparticles as kinetic energy of electrons and holes and the coulomb interaction energy nullify each other.

Table 1-Calculated values of Effective band Gap, Kinetic energy and Coulomb energy for various sizes of CdS nanoparticles

\begin{tabular}{|l|l|l|l|l|}
\hline $\begin{array}{l}\boldsymbol{R}_{\mathbf{0}} \\
(\mathbf{n m})\end{array}$ & $\begin{array}{l}\boldsymbol{E}\left(\boldsymbol{R}_{\mathbf{0}}\right) \\
(\mathbf{e V})\end{array}$ & $\begin{array}{l}\text { Wavelength } \\
(\mathbf{n m})\end{array}$ & $\begin{array}{l}\text { Kinetic } \\
\text { Energy } \\
\text { Term (eV) }\end{array}$ & $\begin{array}{l}\text { Coulomb } \\
\text { Energy } \\
\text { Term (eV) }\end{array}$ \\
\hline 1 & 4.42 & 280 & 2.44 & 0.45 \\
\hline 1.5 & 3.21 & 385 & 1.08 & 0.30 \\
\hline 2 & 2.81 & 440 & 0.61 & 0.23 \\
\hline 3 & 2.55 & 485 & 0.27 & 0.15 \\
\hline 4 & 2.47 & 501 & 0.15 & 0.11 \\
\hline 5 & 2.44 & 508 & 0.10 & 0.09 \\
\hline
\end{tabular}

(Parameters: Bohr's exciton radius $=3 \mathrm{~nm}$ [18], Effective mass $m^{*}{ }_{h}=0.19 m_{0}$ and $m^{*}{ }_{e}=0.8 m_{0}$ and Dielectric constant $\left.\varepsilon_{r}=5.7\right)$

Table 2 - Calculated values of Effective band Gap, Kinetic energy and Coulomb energy for various sizes of $\mathrm{ZnS}$ nanoparticles

\begin{tabular}{|l|l|l|l|l|}
\hline $\begin{array}{l}\boldsymbol{R}_{\mathbf{0}} \\
(\mathbf{n m})\end{array}$ & $\begin{array}{l}\boldsymbol{E}\left(\boldsymbol{R}_{\mathbf{0}}\right) \\
(\mathbf{e V})\end{array}$ & $\begin{array}{l}\text { Wavelength } \\
(\mathbf{n m})\end{array}$ & $\begin{array}{l}\text { Kinetic } \\
\text { Energy } \\
\text { Term (eV) }\end{array}$ & $\begin{array}{l}\text { Coulomb } \\
\text { Energy } \\
\text { Term (eV) }\end{array}$ \\
\hline 1 & 4.90 & 253 & 1.53 & 0.31 \\
\hline 1.5 & 4.15 & 298 & 0.68 & 0.21 \\
\hline 2 & 3.91 & 317 & 0.38 & 0.16 \\
\hline 3 & 3.75 & 330 & 0.17 & 0.10 \\
\hline 4 & 3.70 & 335 & 0.10 & 0.08 \\
\hline
\end{tabular}

(Parameters: Bohr's exciton radius $=2.5 \mathrm{~nm}$ [19], Effective mass $m_{h}^{*}=0.61 m_{0}$ and $m_{e}^{*}=0.41 m_{0}$ and Dielectric constant $\varepsilon_{r}=8.3$ )

The plot for normalized PL intensity vs photon energy are shown in Figure 2 and Figure 3 for monodisperse CdS and ZnS quantum dots. It can be seen that the shape of the emission peak is same, only there is shift towards lower energy or larger wavelength by increasing particle radius. In case of CdS nanoparticles of radius $4 \mathrm{~nm}$ or larger than this there is overlapping and very little shifting of peaks. Similar overlapping and shifting of peaks were observed for $\mathrm{ZnS}$ nanoparticles of radius greater than $3 \mathrm{~nm}$.

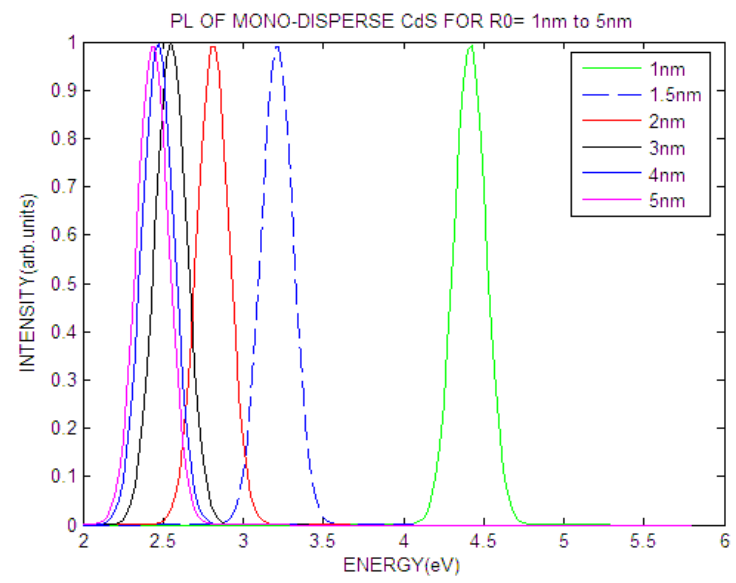

Fig. 2 - Normalized intensity vs Photon energy

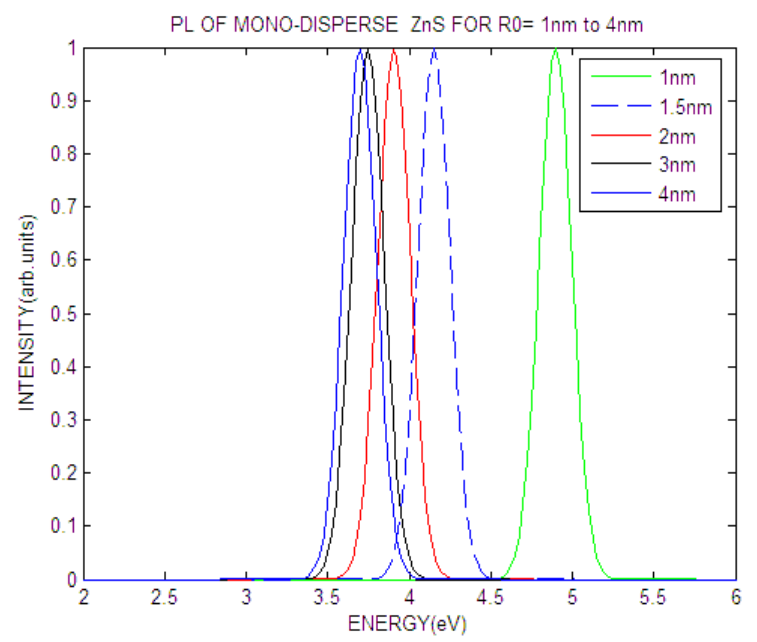

Fig. 3 - Normalized intensity vs Photon energy 


\section{DISCUSSION AND EXPERIMENTAL SUPPORT}

Many researchers have reported luminescence of nanocrystals of various materials. Their results support the theory described above. The comparison of experimental results and theory is discussed here. The systematic investigation of II-VI quantum dots $(\mathrm{ZnS}, \mathrm{CdS}$, CdSe, etc.) began using solution-phase synthesis [20]. It also was inspired by a theoretical work which showed the linear and resonant nonlinear optical properties exhibit the greatest enhancement when the nanoparticle radius $R$ is much smaller than the Bohr radius of the exciton $\left(a_{B}\right)$ in the corresponding bulk material [21]. Deviation of properties are expected for semiconductor QDs in the strongconfinement limit, where $R<a_{B}$. The Bohr radii of excitons vary strongly between different semiconductors: $10 \AA$ (CuCl), $60 \AA$ (CdSe), $200 \AA$ (PbS), $340 \AA$ (InAs), $460 \AA$ (PbSe), $540 \AA$ (InSb) [22]. In contrast, the energy level structure of II-VI and III-V QDs are much more complicated, with closely spaced hole levels and valence band mixing which complicates the study of these materials.

SN Sahu and KK Nanda reported about the CdS thin film sample of different crystalline sizes and thickness was prepared by chemical route method and found the band gap of CdS increases from $2.5 \mathrm{eV}$ to $4.5 \mathrm{eV}$ for decreasing particle size from $6 \mathrm{~nm}$ to $1 \mathrm{~nm}$ [23]. This is in agreement with the theory described in the present work.

Wageh et al [24] have studied optical properties of mercapto acitic acid capped $\mathrm{ZnS}$ nanoparticles. They have prepared $\mathrm{ZnS}$ nanoparticles of size $3.5 \mathrm{~nm}$ and $4.4 \mathrm{~nm}$ as obtained by XRD and their effective band gap from the absorption spectra is found to be $4.27 \mathrm{eV}$ and $4.05 \mathrm{eV}$ respectively. The theory modeled here gives the values of effective band gaps of $\mathrm{ZnS}$ nanoparticles of particle sizes $\left(2 R_{0}\right) \quad 3.5 \mathrm{~nm}$ and $4.4 \mathrm{~nm}$ (where $R_{0}=1.75 \mathrm{~nm}$ and $2.2 \mathrm{~nm}$ radius) as $4.1 \mathrm{eV}$ and $3.9 \mathrm{eV}$ respectively. These are quite close to the experimentally observed results of Wageh et al.

\section{CONCLUSIONS}

Many workers have reported band to band PL for a number of materials. In case of nanocrystals the PL

\section{REFERENCES}

1. M. Verma, D. Patidar, K.B. Sharma, N.S. Saxena, J. Nanoelectron. Optoelectron. 10, 320 (2015).

2. M. Ramrakhiani, V. Nagoriya, J. Lumin. 133, 129 (2013).

3. Z. Zhu, et al., Phys. Chem. Chem. Phys. 15, 1279 (2013).

4. P. Hari Krishna, M. Ramrakhiani, AIP Conf. Proc. 2270 , 110039 (2020).

5. S. Bhanoth, PV. More, A. Jadhav, P.K. Khanna, RSC Adv. 4, 17526 (2014).

6. Botao Ji, S. Koley, Ilya Slobodkin, S. Remennik, Uri Banin, Nano Lett. 20, 2387 (2020).

7. Y.E. Panfil, M. Oded, U. Banin, Angew. Chem., Int. Ed. 57, 4274 (2018)

8. L.E. Brus, J. Chem. Phys. 79, 5566 (1983).

9. N. Karar, F. Singh, B.R. Mehta, J. Appl. Phys. 95, 656 (2004).

10. T.R. Ravindran, A.K. Arora, B. Balamurugan, B.R. Mehta, Nanostructur. Mater. 14 No 5, 603 (1999).

11. H. Wei, Y. Su, S. Chen, Y. Liu, Y. Lin, Y. Zhang, Mater. Lett. 67 No 1, 269 (2012).

12. L.E. Brus, J. Chem. Phys. 80, 4403 (1984).

13. D.C. Onwudiwe, P.A. Ajibade, Int. J. Molec. Sci. 12 No 9, 5538 (2011). peak is observed at same energy as the absorption edge which shifts towards shorter wavelength for smaller particles. Here the effect of particle size of semiconductor nanoparticles on the band-edge spectrum is investigated theoretically. The important conclusions drawn from the studies of the effect of particle size on emission wavelength are as given below:

1. The PL spectra of bulk material is similar in all cases when similar vibronic coupling and normalized PL intensity is considered only the peak wavelength changes for $\mathrm{CdS}, \mathrm{ZnS}, \mathrm{ZnSe}$ and CdSe as $510 \mathrm{~nm}, 337 \mathrm{~nm}, 441 \mathrm{~nm}$ and $724 \mathrm{~nm}$ respectively corresponding to their band gap.

2. The variation in band gap may be obtained by reducing the particle size to very small values $<2$ exciton radii. This also shifts the band edge luminescence peak to higher energies.

3. It is also observed that the PL peak photon energy or the effective band gap for nanoparticle of $1 \mathrm{~nm}$ radius is quite large for small $E_{g}$ material. The increase in effective band gap depends not only on the bulk band gap but also on effective masses of holes and electrons , dielectric constants and exciton Bohr radius. Hence by reducing nanocrystal size, small band gap substance can be used to obtain visible light.

4. At the end we can conclude when dispersion is zero and particle size decreases there is only shift in the PL peak position with no broadening. Similar results may be observed in $\mathrm{ZnSe}$ and CdSe nanoparticles too.

\section{ACKNOWLEDGEMENTS}

The authors would like to thank retired professors Dr. Laxman Pandey Rani Durgawati University Jabalpur, Madhya Pradesh for motivation and guidance. In queue, I would also show my gratitude to Dr. Malyaj Das, Head of Department Physics and Prof. (Dr.) Sunil K. Somani, Vice Chancellor, Medi-Caps University, Indore, Madhya Pradesh for assisting and providing mathematical tools, software needed for the research. Lastly, I would give my regards to Dr. Jitendra Tripathi, Head of the Department Physics, ISR and Dr. Archana Keerti Chowdhary, Principal, IPS Academy, IES, Indore for the encouragement and support.

14. V. Kumar, A.K. Shrivastava, A. Sinha, V. Jha, Indian J. Pure Appl. Phys. 51 No 1, 49 (2013).

15. J.F. Wang, A. Omino, M. Isshiki, Mater. Sci. Eng. B 83 No 1-3, 185 (2001).

16. S.H. Song, J.F. Wang, G.M. Lalev, L. He, M. Isshiki, J. Crystal Growth 252 No 1-3, 102 (2003).

17. Mönch Winfried, Electronic Properties of Semiconductor Interfaces (Springer-Verlag Berlin Heidelberg: 2004).

18. S.N. Sahu, K.K. Nanda, PINSA 67, A No 1, 103 (2001).

19. P.E. Lippens, M. Lannoo, Mater. Sci. Eng. B 9 No 4, 485 (1991).

20. N. Chestnoy, T.D. Harris, R. Hull, L.E. Brus, J. Phys. Chem. 90, 3393 (1986).

21. S. Schmitt-Rink, D.S. Miller, D.S. Chemla, Phys. Rev. B 35, 8113 (1987).

22. D.J. Norris, A. Sacra, C.B. Murray, M.G. Bawendi, Phys. Rev. Lett. 72, 2612 (1994).

23. K.K. Nanda, S.N. Sarangi, S. Mohanty, S.N. Sahu, Thin Solid Films 322, 21 (1998).

24. Wageh, et al., J. Lumin. 102-103, 768 (2003). 